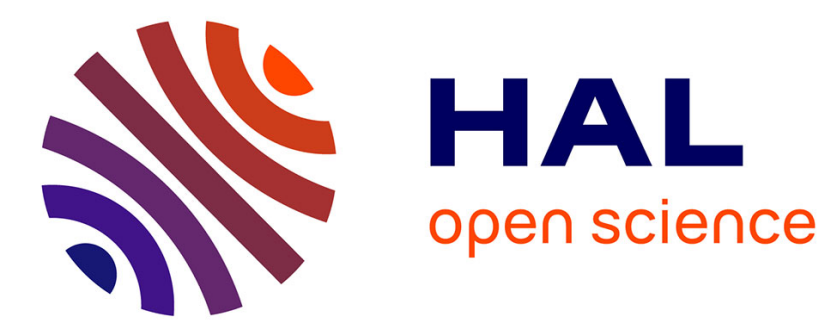

\title{
Piston position estimation for an electro-pneumatic actuator at standstill
}

Frédéric Abry, Xavier Brun, Michaël Di Loreto, Sylvie Sesmat, Eric Bideaux

\section{To cite this version:}

Frédéric Abry, Xavier Brun, Michaël Di Loreto, Sylvie Sesmat, Eric Bideaux. Piston position estimation for an electro-pneumatic actuator at standstill. Control Engineering Practice, 2015, 41 (8), pp.176-185. 10.1016/j.conengprac.2015.04.016 . hal-01256427

\section{HAL Id: hal-01256427 \\ https://hal.science/hal-01256427}

Submitted on 23 Apr 2019

HAL is a multi-disciplinary open access archive for the deposit and dissemination of scientific research documents, whether they are published or not. The documents may come from teaching and research institutions in France or abroad, or from public or private research centers.
L'archive ouverte pluridisciplinaire HAL, est destinée au dépôt et à la diffusion de documents scientifiques de niveau recherche, publiés ou non, émanant des établissements d'enseignement et de recherche français ou étrangers, des laboratoires publics ou privés.

$$
\text { Copyright }
$$




\title{
Piston position estimation for an electro-pneumatic actuator at standstill
}

\author{
ABRY Frédéric, BRUN Xavier, DI LORETO Michael, SESMAT Sylvie and BIDEAUX Éric ${ }^{a}$ \\ ${ }^{a}$ Université of Lyon, INSA - Lyon, Laboratoire Ampère CNRS UMR 5005, \\ 25 avenue Jean Capelle, 69621 Villeurbanne, France \\ frederic.abry@gmail.com
}

\begin{abstract}
In this paper, a first strategy to reconstruct the piston position of a pneumatic cylinder supplied by two servovalves is proposed using only the chambers pressure measurements. This work focuses on the position observation at standstill. Starting from a classical model of this actuator, a transformation of the inputs is used. This way, for the first time, a link is established between electropneumatic actuators and Permanent Magnet Synchronous Motors (PMSM). This allows to apply PMSM zero speed observation strategies to electro-pneumatic actuators. Thus, following an observability analysis, a signal injection based methodology is used to ensure the observability of the electro-pneumatic system. A non-linear position observer is synthesized and a Lyapunov function is provided to ensure the global stability. Experimental results confirm the proposed strategy efficiency. The proposed technique constitutes a first step toward sensorless position control of electro-pneumatic actuators.
\end{abstract}

Keywords: Estimation, observability analysis, nonlinear observer synthesis, electro-pneumatic actuator, signal injection

\section{Introduction}

Over the past decade, reducing the number of sensors used in industrial applications has been one of the major preoccupations of the control community. Sensors can be expensive, not reliable, they can drift over time, and they may not be able to withstand some extreme conditions (humidity, temperature, vibrations...). In any case, whenever it can be safely done, any engineer will replace a physical sensor by a software equivalent. Observers can also be used to improve the reliability of a system by introducing a redundancy in the measurement which allows fault detection and diagnostics.

In particular, control of Permanent Magnets Synchronous Motors (PMSM) without angular position sensor is a subject which has been widely addressed in the literature. It has been shown [1] that PMSM's rotor position is only observable for non-zero speed. Therefore, many position observers have initially been proposed for velocity control applications. The most efficient proposals include the extended Kalman filtering [2] and the sliding mode observers [3]. Those strategies cannot provide the position at zero speed but, once the rotor is in motion, using the current measurements they give a very good estimate of the position and velocity which can then be used for magnetic field orientation and velocity regulation. More recently, propositions have been made to synthesize position observer for PMSMs at zero speed. To overcome the non observability issue, most strategies rely on the injection of a voltage in order to make the system observable $[4,5]$.

While so many propositions have been made in the literature regarding PMSMs position observation, observer designs for electro-pneumatic or electro-hydraulic applications only focus on replacing one of the pressure sensors by an observer [6, 7]. Robust differentiation technique [8] have also been used to re- place the accelerometer or the differential pressure feedback by an acceleration estimation. Yet, so far, no proposition has been made regarding the actual position observation. The algorithm proposed in this paper is therefore very different: the pressure measurements are used to reconstruct the piston position in the same way that the stator currents measurements are used to reconstruct the rotor angular position in PMSMs observer design.

In this paper, using the A-T transformation introduced for the first time in [9], a similar approach is proposed to synthesize a non-linear observer for zero speed reconstruction of the piston position of an electro-pneumatic cylinder. The use of the A-T transformation, which is similar to the d-q Park Transform [10] widely used in three-phase electrical systems control, allows a parallel between electro-pneumatic actuators and PMSMs to be drawn. Therefore, an analogous signal injection method is proposed to make the system observable.

After a short presentation of the model of the electropneumatic actuator generally used for control synthesis purposes, the principle of the A-T transformation is described and compared to the Park Transform. Then, an observability analysis under zero speed condition is conducted. The results are used to define a signal injection strategy consistent with a zero speed observer requirements. A non-linear observer is then proposed. Finally, experimental results are provided to illustrate the efficiency of the algorithm.

\section{Electro-pneumatic actuator description and model}

\subsection{System overview}

An electro-pneumatic actuator (or servo-pneumatic actuator) is the combination of an electric system, used to precisely con- 
trol the gas mass flow rates and a pneumatic system which uses the energy of the pressurized gas to generate a force.

The pneumatic actuator (see Fig.1) used in this study is a compact ASCO Numatics double acting symmetric pneumatic cylinder. Each of its chambers is supplied by an independent servovalve (Festo MPYE 5 1/8 HF). Two miniature sensors are integrated to monitor the pressures in the cylinder chambers.

\begin{tabular}{cc}
\hline Total stroke & $50 \mathrm{~mm}$ \\
\hline Piston diameter & $100 \mathrm{~mm}$ \\
\hline Rod diameter & $28 \mathrm{~mm}$ \\
\hline Piston effective section & $7238 \mathrm{~mm}^{2}$ \\
\hline Maximum force at 7 bar & $4343 \mathrm{~N}$ \\
\hline
\end{tabular}

Table 1: Cylinder main characteristics
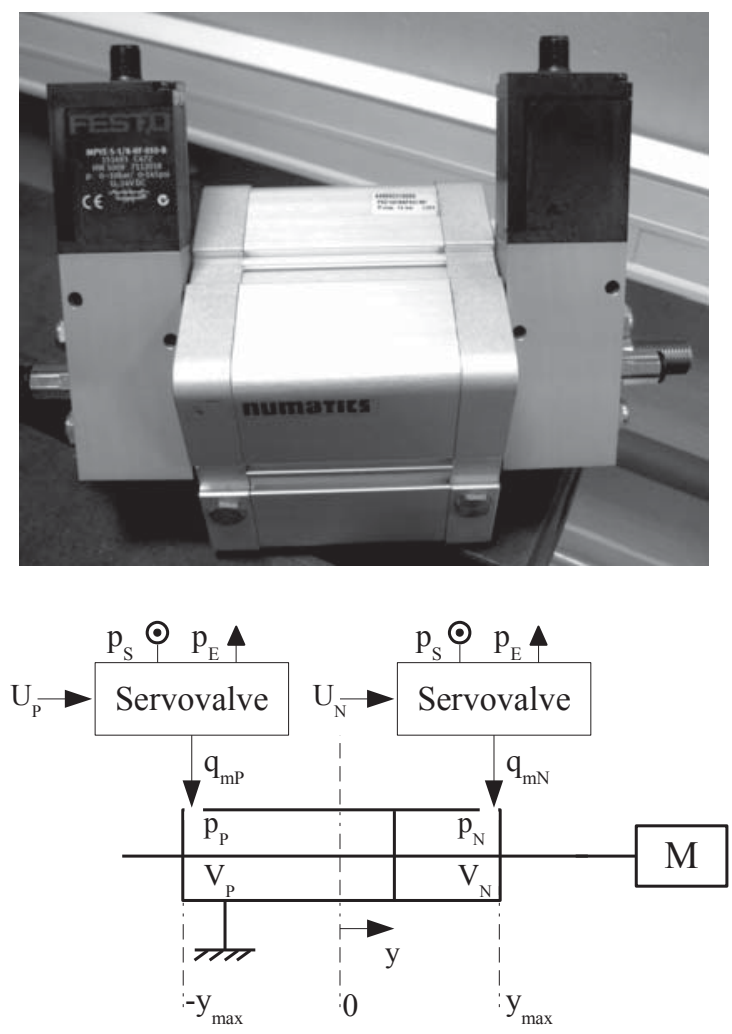

Figure 1: Physical system under consideration

The servovalves are supplied with air at 7 absolute bar through a pressure regulator and a 40 liters buffer tank.

\subsection{Model for control synthesis}

In order to accurately describe the thermodynamical behavior of the cylinder, a model based on fluid mass and energy conservation laws, which takes into account heat exchanges by convection is commonly used in the literature [11, 12]. It is based on the following assumptions: 1)homogeneous temperature and pressure distributions in the chambers, 2)absence of leakage, 3)air is an ideal gas and 4)air kinetic and potential energies are negligible compared to internal energy. It leads to a high order model suitable for simulation purposes but which requires the temperatures measurement to be used as a control model. Thus, a simpler yet fairly accurate representation is generally chosen for control synthesis. The classical way to simplify the model is to adopt a polytropic law and consider the relative temperature variations in both chambers to be small and therefore negligible. This leads to the following reduced order model [13]:

$$
\left\{\begin{aligned}
\frac{d p_{P}}{d t} & =\frac{k r T}{V_{P}(y)}\left(q_{m P}-\frac{S}{r T} p_{P} v\right) \\
\frac{d p_{N}}{d t} & =\frac{k r T}{V_{N}(y)}\left(q_{m_{N}}+\frac{S}{r T} p_{N} v\right)
\end{aligned}\right.
$$

with $v$ the velocity of the piston, defined as the time derivative of the piston position $y, T$ the supply temperature, $r$ the specific ideal gas constant of air, $q_{m P}$ and $q_{m_{N}}$ the mass flow rates defined as positive entering the chambers $\mathrm{P}$ and $\mathrm{N}$ respectively, $V_{P}(y)$ and $V_{N}(y)$ the respective volumes of the $\mathrm{P}$ and $\mathrm{N}$ chambers, $S$ the piston effective surface and $k$ the polytropic coefficient chosen experimentally. The chambers volumes are computed as follows:

$$
\left\{\begin{array}{l}
V_{P}(y)=V_{0}+S y \\
V_{N}(y)=V_{0}-S y
\end{array}\right.
$$

with $y$ the piston position (defined as zero in central position) and $V_{0}$ the cylinder half volume which can be computed as:

$$
V_{0}=S \frac{l}{2}
$$

where $l$ stands for the total cylinder length which includes the dead lengths at each extremity. This ensures that $V_{0}>S\left|y_{\max }\right|$ (where $y_{\max }$ stands for the cylinder's half stroke as defined in Fig.1) and therefore that $V_{P}(y)$ and $V_{N}(y)$ are strictly positive.

The pneumatic force created by the cylinder is:

$$
F_{\text {pпеи }}=S\left(p_{P}-p_{N}\right) .
$$

Thus, the mechanical behavior of the system can be described by:

$$
\left\{\begin{array}{l}
\frac{d v}{d t}=\frac{1}{M}\left[F_{\text {pneu }}-b v-F_{d r y}(v)\right] \\
\frac{d y}{d t}=v
\end{array}\right.
$$

with $M$ the carried mass, $b$ the total cylinder and load viscous friction coefficient and $F_{d r y}(v)$ a function of the velocity representing the dry friction phenomenon. It is often modeled by the well-known Tustin model [14, 15]:

$$
F_{d r y}(v)= \begin{cases}\operatorname{sign}(v)\left[F_{c}+\left(F_{s}-F_{c}\right) e^{-\frac{v}{v_{s}}}\right] \quad \text { if }|v| \neq 0, \\ \sum F & \text { if } v=0 \text { and }\left|\sum F\right|<F_{s}, \\ F_{s} \cdot \operatorname{sign}\left(\sum F\right) & \text { otherwise }\end{cases}
$$

where $\sum F$ is the sum of the forces applied on the piston, $F_{s}$ the static force, $F_{c}$ the Coulomb force and $v_{s}$ the Stribeck 
velocity. The sign function is defined as follows:

$$
\operatorname{sign}(x)= \begin{cases}1 & \text { if } x>0 \\ 0 & \text { if } x=0 \\ -1 & \text { if } x<0\end{cases}
$$

The dry friction phenomenon is known to be very important when compared to the nominal pneumatic force in most electropneumatic system [16]. In most applications, this is a difficult problem to overcome but in the proposed study, the stiction phenomenon is actually preventing the displacement of the piston during the position observation. A more detailed analysis is provided in section 4 .

\subsection{Servovalve inverse model}

In (1), the system's two inputs are the servovalves mass flow rates $q_{m P}$ and $q_{m_{N}}$. Considering the supply and exhaust pressures to be constant, the mass flow rate depends on both the control voltage and the pressure of the chamber supplied. Since the actual output of the control law has to be the servovalves control voltages, an inverse model of the servovalves is required.

In pneumatic applications, the servovalves dynamics can be neglected since they are supposed to be very fast compared to the pressure dynamics in the cylinder. Therefore, only the steady state flow behavior of the servovalve have to be modeled [17].

Mostly two distinct strategies are proposed in the literature, the first one consists in using a theoretical model $[18,12]$ which parameters are then estimated using the supplier data sheet or by experimental tests. The second technique consists in a thorough experimental evaluation of the servovalve mass flow rate for different voltage controls and chamber pressures [19, 20]. From the results is derived a three dimensional table giving the mass flow rate for each couple pressure - control voltage. This table is then used to compute the control voltage [9]. In this study, this last strategy has been chosen.

This experimental inverse model allows the synthesis of control laws taking the mass flow rates as inputs of the system.

\section{The A-T transformation}

\subsection{Introduction: the Park transform}

The A-T transformation proposed for the first time in [9], is similar to the d-q Park Transform [10] widely used in threephase electric motors control. Those systems normally display three inputs: the three voltages $V_{a}, V_{b}$ and $V_{c}$ which control the three currents $I_{a}, I_{b}$ and $I_{c}$. Therefore, the system should offer three degrees of freedom. Yet, since the electric system has to be balanced, a first constraint is imposed: $V_{a}+V_{b}+V_{c}=0$. Thus, the system has only two degrees of freedom.

In the non-salient poles PMSM case for instance, the voltages are applied on the stator windings which produce a magnetic field. The main purpose of control is to synchronize the rotor position and the magnetic field orientation. Physically, the latter can be divided into two components. One is perpendicular to the permanent magnet magnetic field: it induces the electromagnetic torque which can put the rotor into motion. The other one, on the contrary, is parallel to the permanent magnet magnetic field: it does not act on the electromagnetic torque but can only reduce or increase the magnetic flux of the permanent magnet.

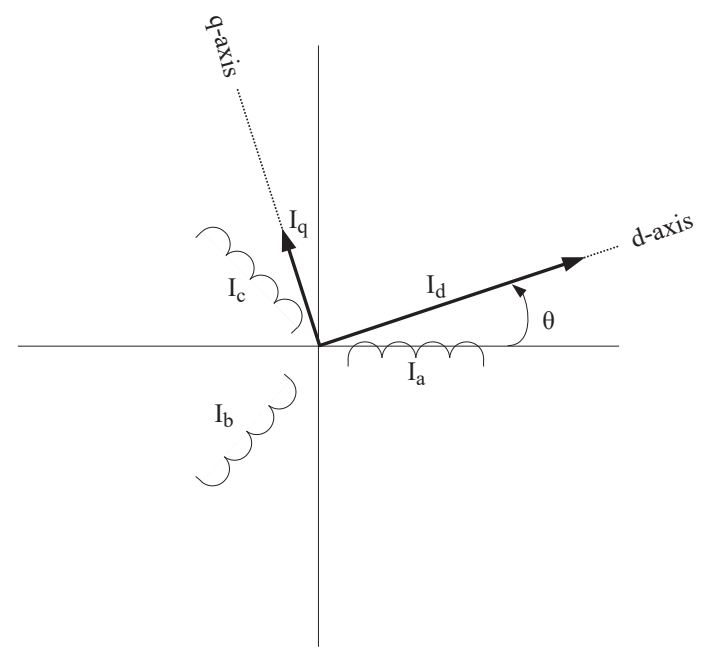

Figure 2: Principle of the Park transform. The three stator currents are expressed in the rotating frame of the rotor.

Using the Park Transform, the three voltages are expressed in the rotor reference frame (see Fig.2) in order to obtain a model which explicitly displays the two degrees of freedom. This is done using the following matrix:

$$
K(\theta)=\sqrt{\frac{2}{3}}\left[\begin{array}{ccc}
\cos (\theta) & \cos \left(\theta-\frac{2 \pi}{3}\right) & \cos \left(\theta+\frac{2 \pi}{3}\right) \\
-\sin (\theta) & -\sin \left(\theta-\frac{2 \pi}{3}\right) & -\sin \left(\theta+\frac{2 \pi}{3}\right)
\end{array}\right]
$$

where $\theta$ stands for the rotor angular position. (8) can then be used to compute the following:

$$
\left[\begin{array}{l}
V_{d} \\
V_{q}
\end{array}\right]=K(\theta)\left[\begin{array}{c}
V_{a} \\
V_{b} \\
V_{c}
\end{array}\right] \text { and }\left[\begin{array}{c}
I_{d} \\
I_{q}
\end{array}\right]=K(\theta)\left[\begin{array}{c}
I_{a} \\
I_{b} \\
I_{c}
\end{array}\right]
$$

Two virtual voltages $V_{d}$ and $V_{q}$ are thus defined. They control respectively the virtual currents $I_{d}$ and $I_{q}$ (which are actually not perfectly decoupled when the rotor is in motion). Using these notations, the electromagnetic torque $T_{e m}$ and flux $\phi_{e m}$ are computed by:

$$
T_{e m}=n_{p} \phi_{f} I_{q}
$$

and

$$
\phi_{e m}=\phi_{f}+L_{d} I_{d}
$$

with $n_{p}$ the number of poles, $\phi_{f}$ the permanent magnet flux and $L_{d}$ the stator inductance on the d-axis.

According to (10) and (11), the electromagnetic flux and torque are respectively function of $I_{d}$ and $I_{q}$. In summary, $V_{d}$ can be used to control the rotor magnetic flux (which is needed for field weakening for instance) and $V_{q}$ to control the torque and, thus, the speed or position of the rotor. 
The objective of the A-T transformation presented in the following section is similar: it is intended to decouple the two degrees of freedom of an electro-pneumatic actuator.

\subsection{Definition of the A-T transformation}

In (1), $q_{m P}$ and $q_{m N}$ have been defined as the system inputs. Each one acts on the respective chamber pressure derivatives $d p_{P} / d t$ or $d p_{N} / d t$ but the main purpose of an electropneumatic actuator is to deliver a pneumatic force $F_{\text {pneu }}$. Its time derivative is obtained by combining equations (1) and (4):

$$
\frac{d F_{\text {pneu }}}{d t}=-k S^{2} v\left(\frac{p_{P}}{V_{P}(y)}+\frac{p_{N}}{V_{N}(y)}\right)+k r T S\left(\frac{q_{m P}}{V_{P}(y)}-\frac{q_{m_{N}}}{V_{N}(y)}\right) .
$$

$F_{\text {pneu }}$ is the first degree of freedom of the system. A physically logical choice for the second one is the average pressure $p_{T}$ which can be computed as:

$$
p_{T}=\frac{p_{P}+p_{N}}{2}
$$

Its time-derivative can be computed as:

$$
\frac{d p_{T}}{d t}=\frac{k S v}{2}\left(\frac{p_{N}}{V_{N}(y)}-\frac{p_{P}}{V_{P}(y)}\right)+\frac{k r T}{2}\left(\frac{q_{m P}}{V_{P}(y)}+\frac{q_{m_{N}}}{V_{N}(y)}\right) .
$$

Both dynamics of $d F_{\text {pneu }} / d t$ and $d p_{T} / d t$ are obviously coupled by the inputs $q_{m P}$ and $q_{m}$. Hence, an alternative choice of inputs, which actually control the critical physical outputs (pneumatic force generation and symmetrical pressurization), can be defined. Therefore, two virtual mass flow rates $q_{m_{A}}$ and $q_{m T}$ are introduced. They can be derived from the actual mass flow rates by means of the following transformation:

$$
\left[\begin{array}{l}
q_{m_{A}} \\
q_{m_{T}}
\end{array}\right]=\boldsymbol{\Lambda}(y)\left[\begin{array}{l}
q_{m P} \\
q_{m_{N}}
\end{array}\right],
$$

with the following transformation matrix:

$$
\boldsymbol{\Lambda}(y)=V_{0}\left[\begin{array}{cc}
\frac{1}{V_{P}(y)} & -\frac{1}{V_{N}(y)} \\
\frac{1}{V_{P}(y)} & \frac{1}{V_{N}(y)}
\end{array}\right]
$$

with

$$
\operatorname{det}(\boldsymbol{\Lambda}(y))=\frac{2 V_{0}}{V_{P}(y) V_{N}(y)} \neq 0, \forall y \in\left[-y_{\max } y_{\max }\right] .
$$

According to (17) the matrix is invertible for any position of the piston.

The time derivatives of $F_{\text {pneu }}$ abd $p_{T}$ are obtained by combining equations (12), (14), (15) and (16):

$$
\frac{d F_{\text {pneu }}}{d t}=-k S^{2} v\left(\frac{p_{P}}{V_{P}(y)}+\frac{p_{N}}{V_{N}(y)}\right)+\frac{k r T S}{V_{0}} q_{m_{A}}
$$

and

$$
\frac{d p_{T}}{d t}=\frac{k S v}{2}\left(\frac{p_{N}}{V_{N}(y)}-\frac{p_{P}}{V_{P}(y)}\right)+\frac{k r T}{2 V_{0}} q_{m T} .
$$

According to equations (18) and (19), $q_{m_{A}}$ only acts on the pneumatic force $F_{\text {pneu }}$. It is therefore called the active mass flow rate. On the other hand, $q_{m T}$ can only induce a symmetrical pressurization, without modifying the pneumatic force and therefore corresponds to the pressurization mass flow rate. Thus, the two virtual inputs defined in (15) actually control two independent behaviors of the electro-pneumatic cylinder: the force generation and the symmetrical pressurization.

The notation "A" refers to the active mass flow rate and " $\mathrm{T}$ " to the symmetrical one. The latter was chosen instead of "S" because $p_{S}$ is usually the standard notation for supply pressure.

At this point, a clear parallel between the A-T and Park transformations can be drawn. In both cases, $q_{m_{A}}$ and $V_{q}$ are the main inputs which control respectively the pneumatic force and the electromagnetic torque. $q_{m_{T}}$ and $V_{d}$ control respectively the average pressure level and the magnetic flux and, therefore, have a less straightforward interest in most control applications. However, for zero speed position observer synthesis, they can both be used to make the system (respectively the electro-pneumatic actuator or the PMSM) observable as shown in section 5 .

\section{Zero speed model}

Choosing $\mathbf{x}=\left[\begin{array}{llll}y & v & F_{\text {pneu }} & p_{T}\end{array}\right]^{T}$ as the new state vector and using the A-T transformation, the model for control synthesis described previously becomes:

$$
\left\{\begin{array}{l}
\frac{d y}{d t}=v \\
\frac{d v}{d t}=\frac{-b v-F_{d r y}(v)+F_{\text {pneu }}}{M} \\
\frac{d F_{\text {pneu }}}{d t}=\frac{A_{1} v y F_{\text {pneu }}-A_{2} v p_{T}}{V_{P}(y) V_{N}(y)}+B_{1} q_{m_{A}} \\
\frac{d p_{T}}{d t}=\frac{-A_{3} v F_{p n e u}+A_{4} v y p_{T}}{V_{P}(y) V_{N}(y)}+B_{2} q_{m T}
\end{array}\right.
$$

with

$$
\begin{array}{lll}
A_{1}=k S^{2} & A_{2}=2 k S^{2} V_{0} & A_{3}=\frac{k V_{0}}{2} \\
A_{4}=k S^{2} & B_{1}=\frac{k r T S}{V_{0}} & B_{2}=\frac{k r T}{2 V_{0}} .
\end{array}
$$

Under the zero speed condition $v=0$, (20) becomes:

$$
\left\{\begin{array}{l}
\frac{d y}{d t}=0 \\
\frac{d v}{d t}=\frac{-F_{d r y}(0)+F_{p n e u}}{M} \\
\frac{d F_{\text {pneu }}}{d t}=B_{1} q_{m_{A}} \\
\frac{d p_{T}}{d t}=B_{2} q_{m T}
\end{array} .\right.
$$


If the speed of the piston is initially zero, for the piston to remain still, the speed derivative $d v / d t$ defined in (22) has to be zero. According to (6), this will be the case as long as the sum of the forces applied on the piston remains lower than the dry friction static force $F_{s}$. Therefore, this is ensured if and only if:

$$
\left|F_{\text {pneu }}\right| \leq F_{s}
$$

If (23) is not satisfied, the zero speed model (22) is no longer valid. It has to be noted that the model (22) does not depend on the dry friction static force and the latter will not affect the precision of the observation strategy proposed in section 6 . Therefore only a lower bound of the dry friction static force is needed in order to ensure the condition (23).

In (22), the inputs are the active and pressurization mass flow rates $q_{m_{A}}$ and $q_{m}$. Yet, since the position of the piston is unknown, the A-T transformation cannot be performed and the value of $q_{m_{A}}$ and $q_{m_{T}}$ cannot be set. Therefore the estimated position $\hat{y}$ has to be introduced as well as the estimated virtual mass flow rates $\hat{q}_{m_{A}}$ and $\hat{q}_{m_{T}}$. They are the result of the A-T transformation computed at the estimated position $\hat{y}$ and they are the output of any control law synthesized when the actual position $y$ is unknown. The electro-pneumatic actuator will therefore receive the following mass flow rates

$$
\left[\begin{array}{l}
q_{m P} \\
q_{m_{N}}
\end{array}\right]=\frac{1}{2 V_{0}}\left[\begin{array}{cc}
V_{P}(\hat{y}) & V_{P}(\hat{y}) \\
-V_{N}(\hat{y}) & V_{N}(\hat{y})
\end{array}\right]\left[\begin{array}{l}
\hat{q}_{m_{A}} \\
\hat{q}_{m_{T}}
\end{array}\right]
$$

where $V_{P}(\hat{y})=V_{0}+S \hat{y}$ and $V_{N}(\hat{y})=V_{0}-S \hat{y}$ are the estimated chamber volumes. In the A-T reference frame, the system will therefore receive the following active and pressurization mass flow rates:

$$
\left[\begin{array}{c}
q_{m_{A}} \\
q_{m T}
\end{array}\right]=V_{0}\left[\begin{array}{cc}
\frac{1}{V_{P}(y)} & -\frac{1}{V_{N}(y)} \\
\frac{1}{V_{P}(y)} & \frac{1}{V_{N}(y)}
\end{array}\right]\left[\begin{array}{l}
q_{m P} \\
q_{m_{N}}
\end{array}\right]
$$

The latter can then be computed combining (24) and (25)

$$
\begin{aligned}
q_{m_{A}} & =\hat{q}_{m_{A}}+\bar{y} \frac{S V_{0} \hat{q}_{m_{T}}-S^{2} y \hat{q}_{m_{A}}}{V_{P}(y) V_{N}(y)} \\
q_{m T} & =\hat{q}_{m_{T}}+\bar{y} \frac{S V_{0} \hat{q}_{m_{A}}-S^{2} y \hat{q}_{m_{T}}}{V_{P}(y) V_{N}(y)}
\end{aligned}
$$

where $\bar{y}$ stands for the position estimation error defined as:

$$
\bar{y}=\hat{y}-y .
$$

As long as the zero speed condition (23) is satisfied, $v=0$ and the state vector can be reduced to $\mathbf{x}_{\mathbf{S}}$ defined as:

$$
\mathbf{x}_{\mathbf{s}}=\left[\begin{array}{ll}
y & F_{\text {pneu }} \\
p_{T}
\end{array}\right]^{T} .
$$

Finally, the state model (22) becomes:

$$
\left\{\begin{array}{l}
\frac{d y}{d t}=0 \\
\frac{d F_{\text {pneu }}}{d t}=B_{1}\left(\hat{q}_{m_{A}}+\bar{y} \frac{S V_{0} \hat{q}_{m_{T}}-S^{2} y \hat{q}_{m_{A}}}{V_{P}(y) V_{N}(y)}\right) . \\
\frac{d p_{T}}{d t}=B_{2}\left(\hat{q}_{m_{T}}+\bar{y} \frac{S V_{0} \hat{q}_{m_{A}}-S^{2} y \hat{q}_{m_{T}}}{V_{P}(y) V_{N}(y)}\right)
\end{array}\right.
$$

Special attention has to be given to the following notations :

- $q_{m_{A}}$ and $q_{m T}$ are the actual active and pressurization mass flow rates. They are the result of the A-T transformation performed at the actual piston position $y$ using the real mass flow rates $q_{m P}$ and $q_{m_{N}}$. Since the position $y$ is not measured in this observation problem, $q_{m_{A}}$ and $q_{m T}$ are unknown and cannot be set by control.

- On the contrary, $\hat{q}_{m_{A}}$ and $\hat{q}_{m_{T}}$ are the estimated active and pressurization mass flow rates. They are defined as the result of the A-T transformation performed at the piston estimated position $\hat{y}$. Therefore, they are the actual inputs of the system when the only available information about the piston position is the estimate $\hat{y}$.

\section{Observability analysis}

Before attempting to synthesize a position observer, one has to find out under what conditions observability is given. It is assumed here that only the pressure measurements are available. They can be used to compute the pneumatic force $F_{\text {pneu }}$ and the average pressure $p_{T}$. Moreover, using one of the many recently proposed robust differentiation techniques such as a sliding mode based differentiator [21] or an algebraic differentiator [22], the time derivatives of the pneumatic force and the average pressure $d F_{\text {pneu }} / d t$ and $d p_{T} / d t$ can be numerically computed in real time. Using the latter and the estimated virtual mass flow rates, the following expression $W$ can be computed:

$$
W=\left(\frac{\frac{d F_{\text {pneu }}}{d t} \hat{q}_{m_{T}}}{B_{1}}-\frac{\frac{d p_{T}}{d t} \hat{q}_{m_{A}}}{B_{2}}\right)\left(\hat{q}_{m_{T}}^{2}-\hat{q}_{m_{A}}^{2}\right) .
$$

Using (29), (30) can be rewritten as:

$$
W=\bar{y} \frac{S V_{0}}{V_{P}(y) V_{N}(y)}\left(\hat{q}_{m_{T}}^{2}-\hat{q}_{m_{A}}^{2}\right)^{2}
$$

$W$ has no physical meaning, it is solely a virtual measurement computed in real time by means of (30) using only the time derivatives of the pneumatic force and average pressure as well as the estimated virtual mass flow rates. The main property of $W$ is that, according to (31), it is the product of the estimation error $\bar{y}$ and a positive value.

The following measurement vector $\mathbf{y}_{\mathbf{m}}$ is chosen: 


$$
\mathbf{y}_{\mathbf{m}}=\left[\begin{array}{c}
F_{\text {рпеи }} \\
p_{T} \\
W
\end{array}\right] .
$$

In this section, the observability of the electro-pneumatic actuator at zero speed (represented by (29)) is studied when only $\mathbf{y}_{\mathbf{m}}$ is available. First, the Jacobian matrix $\mathbf{J}_{\mathbf{m}}$ has to be defined:

$$
\mathbf{J}_{\mathbf{m}}=\frac{d \mathbf{y}_{\mathbf{m}}\left(\hat{y}, y, F_{\text {pneu }}, p_{T}, \hat{q}_{m_{A}}, \hat{q}_{m_{T}}\right)}{d \mathbf{x}_{\mathbf{s}}}
$$

where $\mathbf{x}_{\mathbf{s}}$ stands for the state vector defined in (28). It leads to

$$
\mathbf{J}_{\mathbf{m}}\left(\hat{y}, y, \hat{q}_{m_{A}}, \hat{q}_{m_{T}}\right)=\left[\begin{array}{ccc}
0 & 1 & 0 \\
0 & 0 & 1 \\
J_{31}\left(\hat{y}, y, \hat{q}_{m_{A}}, \hat{q}_{m_{T}}\right) & 0 & 0
\end{array}\right]
$$

where:

$$
\begin{aligned}
J_{31} & =\frac{\partial W}{\partial y} \\
& =-\frac{S V_{0}\left(\hat{q}_{m_{T}}^{2}-\hat{q}_{m_{A}}^{2}\right)^{2}\left(V_{0}^{2}+y^{2} S^{2}-2 \hat{y} y S^{2}\right)}{\left(V_{P}(y) V_{N}(y)\right)^{2}} .
\end{aligned}
$$

It has to be noted that in equation (35) the estimated position $\hat{y}$ is algebraically independent of the real position $y$ since the latter is unknown. For $\mathbf{J}_{\mathbf{m}}$ to be of full rank, and, therefore, the system to be locally weakly observable (see Theorem 3.1 in [23]), $J_{31}$ needs to be non-zero. Therefore, the condition for local weak observability is:

$$
J_{31} \neq 0
$$

which is equivalent to

$$
V_{0}^{2}+y^{2} S^{2}-2 \hat{y} y S^{2} \neq 0
$$

and

$$
\hat{q}_{m_{A}}^{2}-\hat{q}_{m_{T}}^{2} \neq 0 .
$$

Since, according to (3), $V_{0}=S \frac{l}{2}$, (37) becomes:

$$
\left(\frac{l}{2}\right)^{2}+y^{2}-2 \hat{y} y \neq 0
$$

The left member of (39) is zero if and only if:

$$
y=\hat{y} \pm \frac{1}{2} \sqrt{\Delta}
$$

with $\Delta=4 \hat{y}^{2}-l^{2}$.

Yet, if the estimated piston position $\hat{y}$, as well as the real piston position $y$, is bounded by the physical limits of the system, that is if $|\hat{y}|<l / 2$ (condition resulting from the existence of dead volumes in both cylinder chambers), then $\Delta$ is strictly negative and there is no real solutions to (39). Therefore the first observability condition (37) is always verified.

The observability condition (38) involves the estimated virtual mass flow rates and can be rewritten as

$$
\left|\hat{q}_{m_{A}}\right| \neq\left|\hat{q}_{m_{T}}\right| .
$$

If this condition is verified then the system is locally weakly observable. In particular, it implies the necessary condition that the system is not observable if both estimated mass flow rates $\hat{q}_{m_{A}}$ and $\hat{q}_{m_{T}}$ are zero (which, according to (24) and (26), is equivalent to $q_{m_{A}}=q_{m T}=0$ and $\left.q_{m P}=q_{m_{N}}=0\right)$. For this reason, in order to synthesize a position observer at zero speed, it is imperative to implement a signal injection strategy on the mass flow rates in order to make the system observable.

The zero speed observability condition is somehow less restrictive than its equivalent in the PMSM case. In the latter, voltage injection is not sufficient to make the system observable, the rotor has to be slightly put into motion for the position to be estimated $[4,5]$. In the electro-pneumatic actuator case, no piston movement is needed: the only requirement is that, at least, one of the estimated mass flow rates has to be non-zero and satisfy the condition (41).

\section{Non-linear observer synthesis}

Zero speed PMSM position observers rely most of the time on high frequency signal injection on the estimated $d$ axis of the Park transform $[4,5]$. As long as an error on the position is committed, part of this signal is actually injected on the real $\mathrm{q}$ axis which results in an electromagnetic torque which generates a slight vibration of the rotor sufficient to estimate the position. A similar strategy is proposed here: a non-zero estimated virtual mass flow rate $\hat{q}_{m_{T}}$ is chosen in order to make the system observable. Then, using the measurement vector $\mathbf{y}_{\mathbf{m}}$ defined by (32), an observer can be synthesized to estimate the piston position. The control algorithm has three main objectives:

- ensure that the system tracks a given average pressure trajectory $p_{T}^{d}$ in order to generate a non-zero $\hat{q}_{m_{T}}$ estimated pressurization mass flow rate, so that the system remains observable;

- cancel the pneumatic force $F_{\text {pneu }}$ to ensure the zero speed condition (23) and avoid any undesired movement of the piston. Therefore, the pneumatic force trajectory is defined by $F_{\text {pneu }}^{d}=0$;

- cancel the position observation error $\bar{y}$.

The first step is to define the pneumatic force error and the average pressure error

$$
z_{1}=F_{\text {pneu }}-F_{\text {pneu }}^{d}=F_{\text {pneu }}
$$

and

$$
z_{2}=p_{T}-p_{T}^{d} .
$$


In order to study the dynamic behavior of three error variables $z_{1}, z_{2}$ and $\bar{y}$, the following Lyapunov function candidate is defined by

$$
V=\frac{1}{2}\left(\left(z_{1}^{2}+z_{2}^{2}\right) V_{P}(y) V_{N}(y)+\bar{y}^{2}\right)
$$

Since $V_{P}(y)$ and $V_{N}(y)$ are strictly positive, $\mathrm{V}$ is strictly positive except for the desired equilibrium $z_{1}=z_{2}=\bar{y}=0$ for which it is zero. Moreover, under the zero speed condition (23), $V_{P}(y)$ and $V_{N}(y)$ are constant. Therefore, the time derivative of the Lyapunov function $\mathrm{V}$ can be computed as follows

$$
\frac{d V}{d t}=\left(z_{1} \frac{d z_{1}}{d t}+z_{2} \frac{d z_{2}}{d t}\right) V_{P}(y) V_{N}(y)+\bar{y} \frac{d \bar{y}}{d t} .
$$

Since the velocity is zero, the position error time derivative is

$$
\frac{d \bar{y}}{d t}=\frac{d \hat{y}}{d t}-\frac{d y}{d t}=\frac{d \hat{y}}{d t}
$$

and equation (45) can be rewritten

$$
\frac{d V}{d t}=\left(z_{1} \frac{d z_{1}}{d t}+z_{2} \frac{d z_{2}}{d t}\right) V_{P}(y) V_{N}(y)+\bar{y} \frac{d \hat{y}}{d t} .
$$

$d \hat{y} / d t$ is the system's third input which must be chosen, together with $\hat{q}_{m_{A}}$ and $\hat{q}_{m_{T}}$, to cancel the three errors $z_{1}, z_{2}$ and $\bar{y}$.

First, the following estimated virtual mass flow rates are chosen

$$
\hat{q}_{m_{A}}=-\frac{C_{1} z_{1}}{B_{1}}
$$

and

$$
\hat{q}_{m_{T}}=\frac{\frac{d p_{T}^{d}}{d t}-C_{2} z_{2}}{B_{2}}
$$

where $C_{1}$ and $C_{2}$ are two strictly positive constants.

Likewise, the estimated position derivative is defined as

$$
\begin{aligned}
\frac{d \hat{y}}{d t}= & \hat{y} S^{2}\left(-C_{1} z_{1}^{2}+\left(\frac{d p_{T}^{d}}{d t}-C_{2} z_{2}\right) z_{2}\right) \\
+ & \frac{B_{2} C_{1} S V_{0} z_{1} z_{2}}{B_{1}}-\frac{B_{1} S V_{0} z_{1}\left(\frac{d p_{T}^{d}}{d t}-C_{2} z_{2}\right)}{B_{2}} \\
& -\lambda W-l S^{2}\left|z_{2} \frac{d p_{T}^{d}}{d t}\right| \operatorname{sign}(W)
\end{aligned}
$$

where $\lambda$ is a strictly positive constant and $W$ is the expression defined in equations (30) and (31). If the observability condition (41) is verified, since $V_{0}, S, V_{P}(y)$ and $V_{N}(y)$ are strictly positive

$$
\operatorname{sign}(W)=\operatorname{sign}(\bar{y}) .
$$

From (47), (48), (49) and (50), the time derivative $d V / d t$ of the previously defined Lyapunov function is

$$
\begin{aligned}
\frac{d V}{d t}= & -\bar{y}^{2}\left[S^{2}\left(C_{1} z_{1}^{2}+C_{2} z_{2}^{2}\right)+\lambda \frac{V_{0} S\left(\hat{q}_{m_{A}}^{2}-\hat{q}_{m_{T}}^{2}\right)^{2}}{V_{N}(y) V_{P}(y)}\right] \\
& -V_{N}(y) V_{P}(y)\left(C_{1} z_{1}^{2}+C_{2} z_{2}^{2}\right) \\
& +\sigma
\end{aligned}
$$

where

$$
\begin{aligned}
\sigma & =S^{2}\left(\bar{y}^{2} \frac{d p_{T}^{d}}{d t} z_{2}-l \bar{y} \operatorname{sign}(\bar{y})\left|\frac{d p_{T}^{d}}{d t} z_{2}\right|\right) \\
& =S^{2}\left(\bar{y}^{2} \frac{d p_{T}^{d}}{d t} z_{2}-l|\bar{y}|\left|\frac{d p_{T}^{d}}{d t} z_{2}\right|\right) .
\end{aligned}
$$

As mentioned in section 5 , both the piston position and estimated position are considered to be bounded by the physical limits. Therefore: $y \in]-l / 2 l / 2[, \hat{y} \in]-l / 2 l / 2[$ and $|\bar{y}|<l$. The latter leads to

$$
\bar{y}^{2} \leq l|\bar{y}| \text {. }
$$

Combining (54) and (53), $\sigma$ can be bounded:

$$
\begin{aligned}
\sigma & \leq S^{2} \bar{y}^{2}\left(\frac{d p_{T}^{d}}{d t} z_{2}-\left|\frac{d p_{T}^{d}}{d t} z_{2}\right|\right) \\
& \leq 0 .
\end{aligned}
$$

Using (52) and (55), the time derivative of the Lyapunov function can be bounded as well by

$$
\begin{aligned}
\frac{d V}{d t} \leq & -\bar{y}^{2}\left[S^{2}\left(C_{1} z_{1}^{2}+C_{2} z_{2}^{2}\right)+\lambda \frac{V_{0} S\left(\hat{q}_{m_{A}}^{2}-\hat{q}_{m_{T}}^{2}\right)^{2}}{V_{N}(y) V_{P}(y)}\right] \\
& -V_{N}(y) V_{P}(y)\left(C_{1} z_{1}^{2}+C_{2} z_{2}^{2}\right) .
\end{aligned}
$$

Therefore, according to (52) and (56), provided that the observability condition (41) is verified, $d V / d t$ is strictly negative except at the desired equilibrium $\bar{y}=z_{1}=z_{2}=0$, therefore the system will asymptoticly track the average pressure trajectory, cancel the pneumatic force and the estimated position $\hat{y}$ will asymptoticly converge to the real position $y$.

It has to be noted that, occasionally, depending on the chosen average pressure trajectory, the observability condition (41) can be not verified. In this case, the time derivative of the Lyapunov function can be zero even if the position observation error is not zero. Therefore, while the system remains unobservable, according to the Lasalle Yoshizawa theorem [24, 25], the position observation error $\bar{y}$ will be bounded and the two errors $z_{1}$ and $z_{2}$ will converge to zero.

Once the estimated virtual mass flow rates $\hat{q}_{m_{A}}$ and $\hat{q}_{m_{T}}$ are computed, the mass flow rates $q_{m_{T}}$ and $q_{m_{N}}$ are calculated using the inverse transformation (24) performed at the estimated position $\hat{y}$ (see Fig.3). Finally the control voltages $U_{P}$ and $U_{N}$ to be applied on the servovalves are computed using the inverse model presented in section 2.3.

\section{Experimental results}

Fig. 4 shows the test bench used to assess the efficiency of the proposed observation algorithm. It combines the electropneumatic actuator described in section 2.1 and a LVDT position sensor (MEAS DC-EC). The latter allows the measurement 


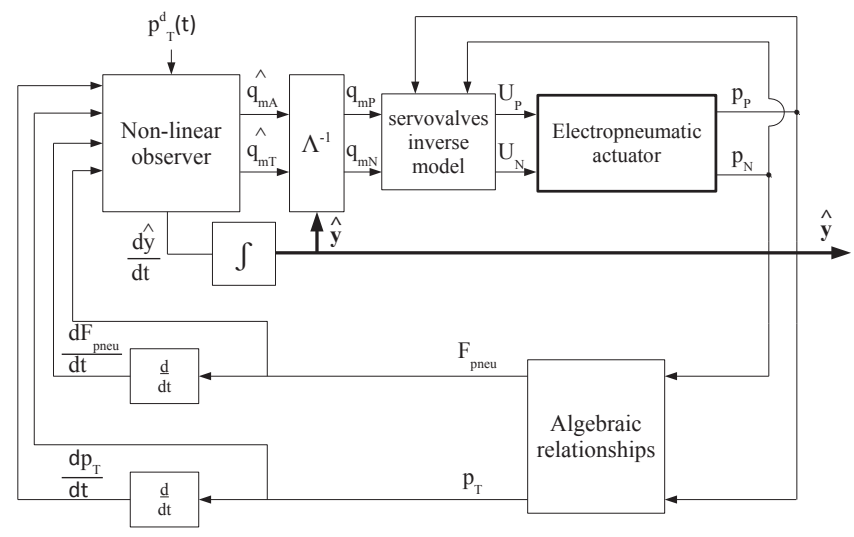

Figure 3: Overview of the observer algorithm.

of the piston displacement which is not used by the observation algorithm but only to assess the precision of the position observation. The test bench also includes a linear electric motor TB30N provided by Tecmotion (included in the experimental setup for other studies). It is only used to move the piston to a given position before the experiment and is therefore absolutely not required for the normal implementation of the observer. Finally, a cRIO-9022 real time controller from National Instruments is used for sensors and actuators interfacing and the rapid prototyping of the control algorithm.

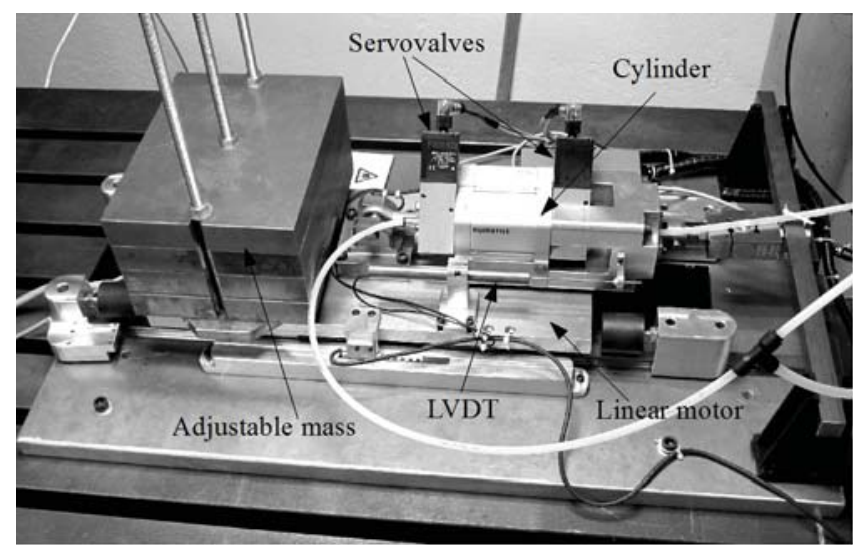

Figure 4: View of the complete test bench

In order to make the system observable, the following average pressure trajectory is chosen:

$$
\begin{aligned}
p_{T}^{d} & =A_{0}+A \sin (\omega t), \\
\frac{d p_{T}^{d}}{d t} & =A \omega \cos (\omega t) .
\end{aligned}
$$

This specific trajectory is chosen because as long as $\bar{y}$ remains non negligible, the tracking of the average pressure trajectory is imprecise and will result in a pneumatic force generation. The tracking of small oscillations by the average pressure can only result in small oscillations of the pneumatic force which are not likely to induce a movement of the piston.
The time derivatives of the pneumatic force and the average pressure $d F_{\text {pneu }} / d t$ and $d p_{T} / d t$ are computed in real time using a sliding mode based adaptive algorithm [26]. Numerical values of the whole control law parameters are summarized in table 2. The amplitude of the average pressure trajectory $A$, its pulsation $\omega$, as well as the observer gain $\lambda$ are chosen experimentally in order to limit the piston displacement and obtain the best tradeoff between convergence time and static precision.

\begin{tabular}{cc}
\hline$C_{1}$ & 10 \\
\hline$C_{2}$ & 50 \\
\hline$\lambda$ & 2 \\
\hline$A_{0}$ & $3 \mathrm{bar}$ \\
\hline$A$ & $0.5 \mathrm{bar}$ \\
\hline$\omega$ & $6 \pi \mathrm{rad} / \mathrm{s}$ \\
\hline
\end{tabular}

Table 2: Algorithm parameters

\subsection{Test 1: reconstruction of the piston position}

The first test describes the process of the piston position estimation for a given initial position. The piston has been moved to a position of $-10 \mathrm{~mm}$ using the linear electric motor and the LVDT sensor which are afterward not used in the observation process. During this preliminary operation, the servovalve control voltages are set so that the cylinders chambers remain at ambient pressure. At $t=0.5 \mathrm{~s}$, the observation algorithm is activated. Fig. 5 shows that the estimated position $\hat{y}$ converges to the real position $y$ (measured by the LVDT) in about $1.5 \mathrm{~s}$.

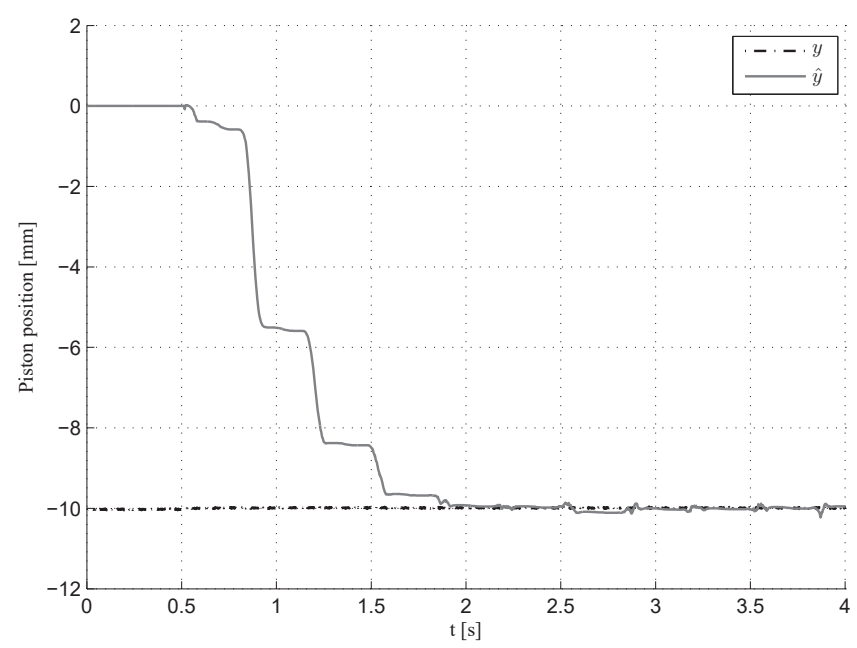

Figure 5: Estimation of the piston position

Fig. 6 shows that the position observation error stays below $0.25 \mathrm{~mm}$ once the steady state is reached which corresponds to $0.5 \%$ of the total stroke. An absolute zero steady state error cannot be achieved because of model errors and measurement noise.

Fig. 7 and 8 respectively show the average pressure trajectory tracking, which ensures the observability of the system, and the pneumatic force. The latter stays below $70 \mathrm{~N}$ and is quickly canceled as the piston position is estimated. This is why the piston remains still during the observation process. 


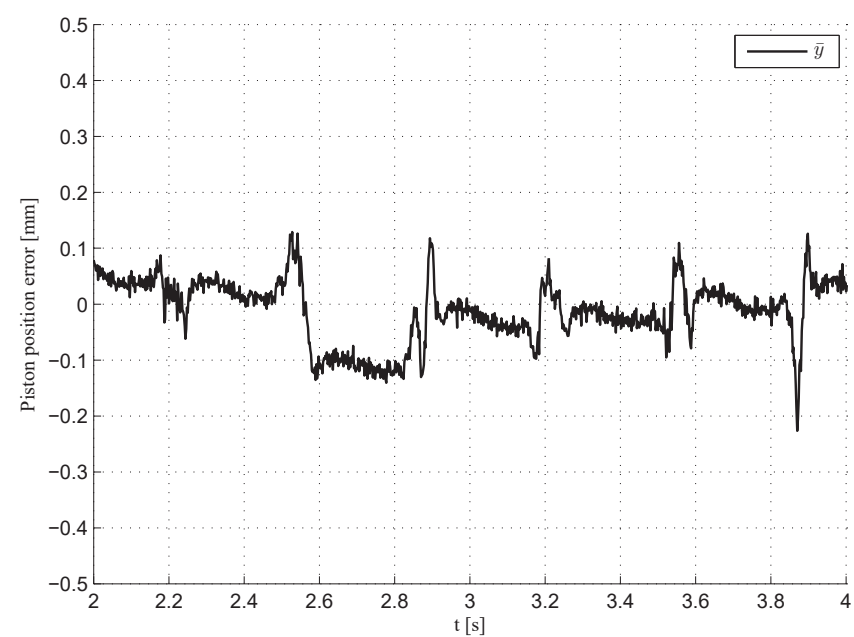

Figure 6: Piston position observation permanent error (zoom)

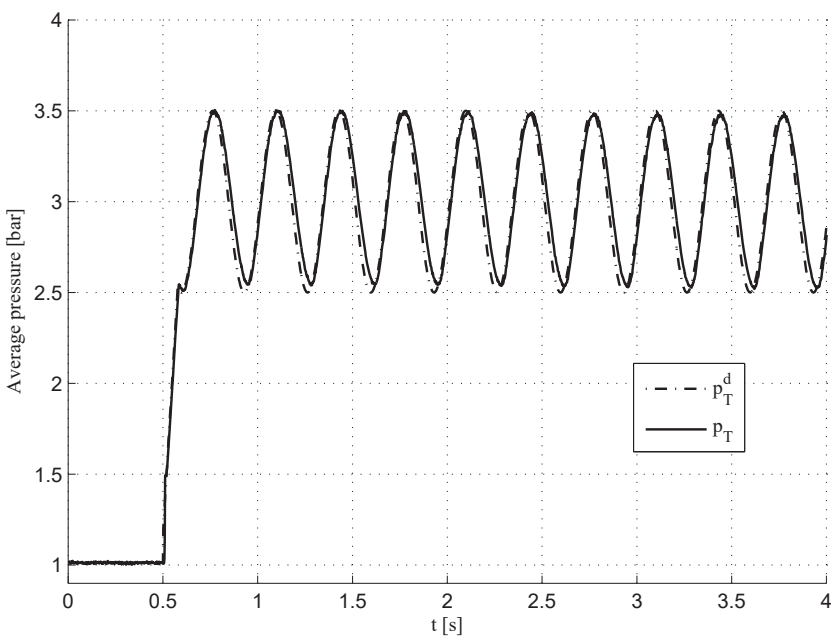

Figure 7: Average pressure trajectory tracking

Fig.10 and 9 respectively show the average pressure and pneumatic force time derivatives.

Finally, Fig. 11 allows a comparative visualization of the evolution of the estimated position $\hat{y}$ and the value of the observability criterion $\hat{q}_{m_{A}}^{2}-\hat{q}_{m_{T}}^{2}$ defined in equation (38). When the latter is zero, the system is not observable, and, according to the study presented in section 6 , the position error $\bar{y}$ should then be bounded. This is verified experimentally since the estimated position $\hat{y}$ remains almost constant while $\hat{q}_{m_{A}}^{2}-\hat{q}_{m_{T}}^{2}$ tends towards zero. On the contrary when the absolute value of the latter increases, the position error $\bar{y}$ converges to zero.

\subsection{Test 2: successive estimations of multiple piston positions}

The second test performed is essentially identical to the first one. The objective is to illustrate the fact that the performances of the observer do not depend on the position. The piston is therefore moved to four different positions using once again the linear electric motor and the LVDT sensor (see Fig.12). During the piston displacement, the observation algorithm is inhibited and the estimated position is reset to zero. Once the piston is

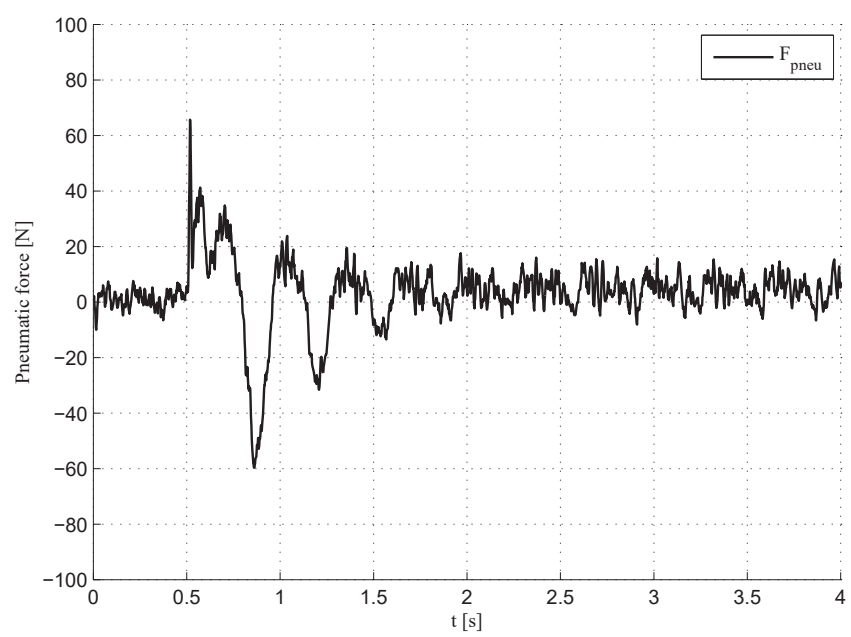

Figure 8: Pneumatic force

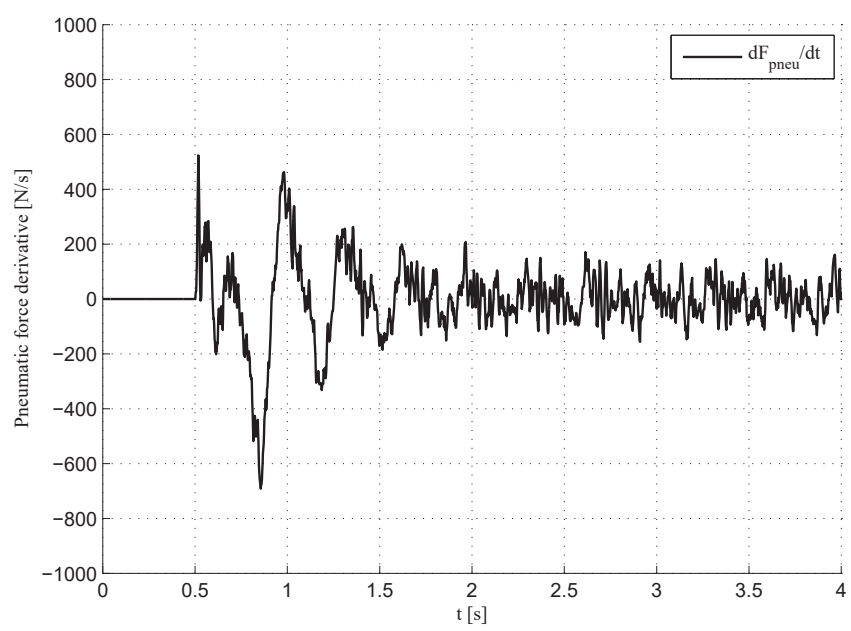

Figure 9: Pneumatic force time derivative

positioned and stands still, the electric motor is inhibited and the observer is activated. In the four cases, the estimated position quickly converges towards the real position (measured by the LVDT sensor).

\subsection{Remark}

It has been observed experimentally that a simpler form of the observation algorithm defined by equations (48), (49) and (58) (which replaces (50)):

$$
\dot{\hat{y}}=-\lambda W
$$

provides experimental results identical to the ones obtained using the algorithm presented in section 6 . However, no theoretical proof has yet been found regarding the convergence of the system thus controlled.

\section{Conclusion}

In this paper, a first study dealing with position observation of an electro-pneumatic actuator was proposed. 


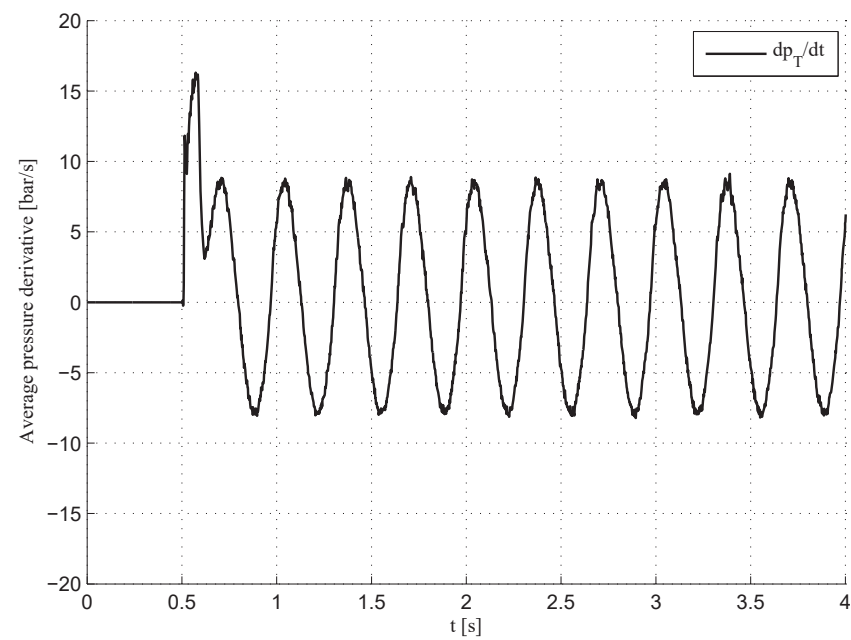

Figure 10: Average pressure time derivative

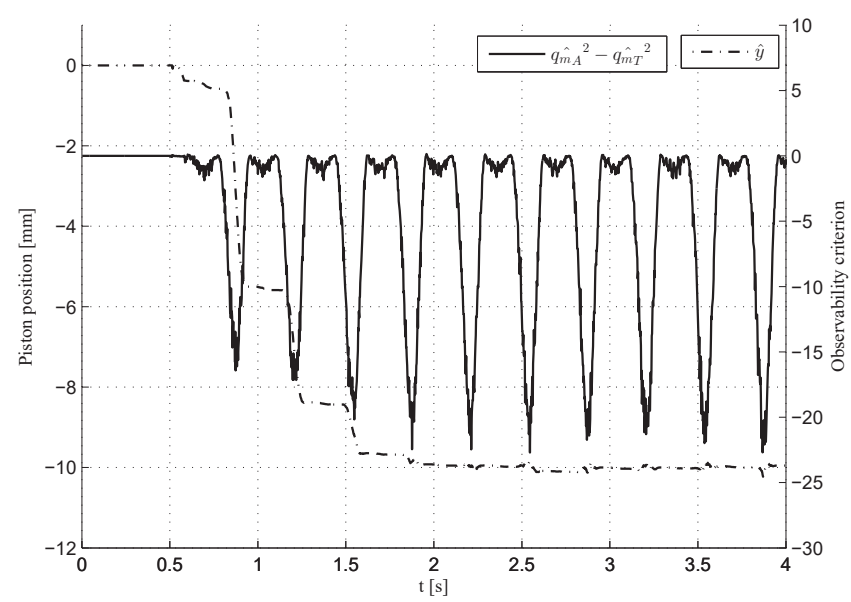

Figure 11: Impact of observability loss over the position observation

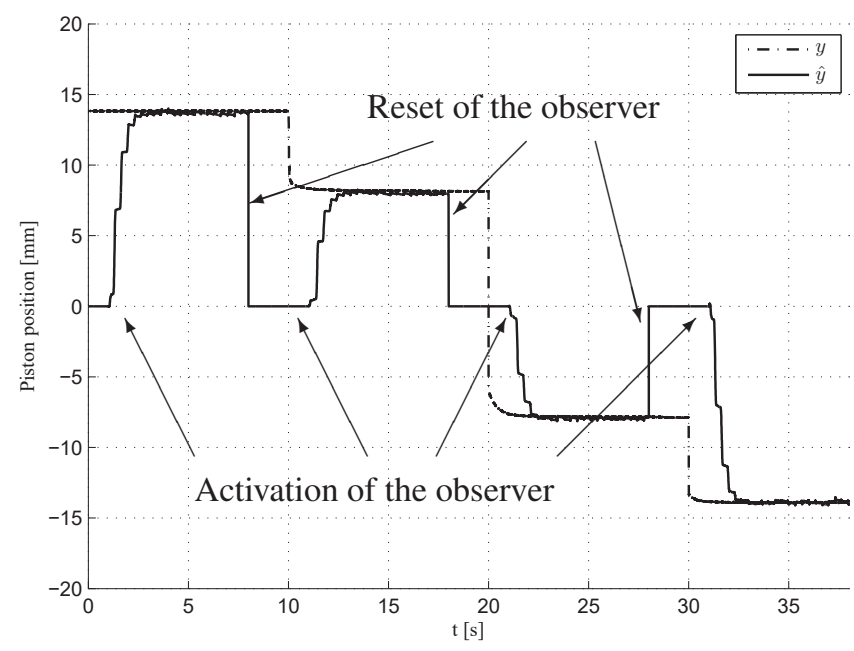

Figure 12: Estimation of the piston position

The use of the A-T transformation allowed to draw a parallel between electro-pneumatic actuators and PMSMs which made easier a transfer of know-how in the field of position observa- tion at zero speed. An observability study was conducted to define the observability conditions at standstill which, similarly to the PMSM case, highlighted the need for a signal injection strategy. The latter was implemented through the tracking of an average pressure trajectory, which, once the position is correctly estimated, does not lead to any movement of the piston. A non-linear observer was then synthesized. A Lyapunov function was defined to demonstrate that the position observation error, as well as the pneumatic force and the average pressure error will asymptoticly converge to zero. Finally, experimental results showed the efficiency of the proposed algorithm. This whole process constitutes a thorough step-by-step example of nonlinear observer synthesis methodology.

This strategy allows a precise reconstruction of the position at standstill: the algorithm can be used to estimate the piston position at zero speed in an open-loop configuration. One possible application is the initialization of a relative sensor (which only measures displacements) such as a magnetoresistive or a an optical coder. Initialization can thus be achieved without inducing any movement of the piston. Other maintenance tasks could also be facilitated by this strategy: imprecise mounting or aging of a sensor can result in a bias or an offset which may have costly consequences. The proposed algorithm offers the possibility to implement a redundant estimation of the position any time the piston is at standstill. This can be done at regular intervals to avoid positioning issues due to a defective sensor.

Beside these practical applications, this work constitutes a first step in the field of position observation of electropneumatic actuators, which, hopefully, will lead in the future to the development of closed-loop sensorless position control laws. Finally, this study also highlights the fact that, due to the significant progress achieved over the last decade, the available robust differentiation algorithms can (and should) be used in observation applications.

\section{References}

[1] D. Zaltni, M.-N. Abdelkrim, M. Ghanes, J. P. Barbot, Observability analysis of pmsm, in: Signals, Circuits and Systems (SCS), 2009 3rd International Conference on, 2009, pp. 1-6.

[2] S. Bolognani, R. Oboe, M. Zigliotto, Dsp-based extended kalman filter estimation of speed and rotor position of a pm synchronous motor, in: Industrial Electronics, Control and Instrumentation, 1994. IECON '94., 20th International Conference on, Vol. 3, 1994, pp. 2097-2102 vol.3.

[3] J. Hu, D. Zhu, Y. Li, J. Gao, Application of sliding observer to sensorless permanent magnet synchronous motor drive system, in: Power Electronics Specialists Conference, PESC '94 Record., 25th Annual IEEE, 1994, pp. 532-536 vol.1.

[4] G. Foo, M. Rahman, Sensorless sliding-mode mtpa control of an ipm synchronous motor drive using a sliding-mode observer and hf signal injection, Industrial Electronics, IEEE Transactions on 57 (4) (2010) 1270_ 1278 .

[5] F. Abry, A. Zgorski, X. Lin-Shi, J.-M. Retif, Sensorless position control for spmsm at zero speed and acceleration, in: Power Electronics and Applications (EPE 2011), Proceedings of the 2011-14th European Conference on, 2011, pp. 1-9.

[6] S. R. Pandian, F. Takemura, Y. Hayakawa, S. Kawamura, Pressure observer-controller design for pneumatic cylinder actuators, Mechatronics, IEEE/ASME Transactions on 7 (4) (2002) 490-499.

[7] N. Gulati, E. J. Barth, A globally stable, load-independent pressure observer for the servo control of pneumatic actuators, Mechatronics, IEEE/ASME Transactions on 14 (3) (2009) 295-306. 
[8] X. Yan, M. Primot, F. Plestan, Comparison of differentiation schemes for the velocity and acceleration estimations of a pneumatic system, in: 19th World Congress The International Federation of Automatic Control, 2014.

[9] F. Abry, X. Brun, S. Sesmat, E. Bideaux, Non-linear position control of a pneumatic actuator with closed-loop stiffness and damping tuning, in: Decision and Control, 2013 European Control Conference. ECC, 2013, pp. 4385-4390.

[10] W. Leonhard, Control of electrical Drives, corrected 2nd printing Edition, Berlin Springer-Verlag, 1990.

[11] H. Jebar, Design of pneumatic actuator systems, Ph.D. thesis, University. Of Nottingham (1977).

[12] P. Beater, Pneumatic drives, Springer, 2007.

[13] A. Chitty, T. Lambert, Modelling a loaded two-way pneumatic actuator, Journal of Dynamic Systems Measurements and Control 9 (1) (1976) 1925.

[14] A. Tustin, The effect of backslash and speed-dependent friction on the stability of closed-cycle control systems, Journal of the institution of electrical engineers 94 (2A) (1947) p143-151.

[15] B. Armstrong-Helouvry, P. Dupont, C. C. De Wit, A survey of models, analysis tools and compensation methods for the control of machines with friction, Automatica 30 (7) (1994) 1083 - 1138.

[16] X. Brun, S. Sesmat, D. Thomasset, S. Scavarda, et al., Study of" sticking and restarting phenomenon" in electropneumatic positioning systems, Journal of Dynamic Systems, Measurement, and Control(Transactions of the ASME) 127 (1) (2005) 173-184.

[17] A. Hildebrandt, O. Sawodny, R. Neumann, A. Hartmann, Cascaded control concept of a robot with two degrees of freedom driven by four artificial pneumatic muscle actuators, in: American Control Conference, 2005. Proceedings of the 2005, 2005, pp. 680-685 vol. 1 . doi:10.1109/ACC.2005.1470036.

[18] D. Mc Cloy, Discharge characteristics of servo valve orifices, in: Fluid Power International conference, no. 6, 1968, pp. 43-503.

[19] A. Wilkening, M. Mihajlov, O. Ivlev, Model-based pressure and torque control for innovative pneumatic soft-actuators, in: Conf. Proc., 7th International Conference on Fluid Power (7th IFK), 2010, pp. 291-302.

[20] M. Belgharbi, S. Sesmat, S. Scavarda, D. Thomasset, Analytical model of the flow stage of a pneumatic servo-distributor for simulation and nonlinear control, The sixth Scandinavian international conference on fluid power (1999) p847-860.

[21] A. Levant, Robust exact differentiation via sliding mode technique, Automatica 34 (3) (1998) 379 - 384.

[22] M. Fliess, H. Sira-Ramírez, An algebraic framework for linear identification, ESAIM: Control, Optimisation and Calculus of Variations 9 (2010) $151-168$.

[23] R. Hermann, A. Krener, Nonlinear controllability and observability, Automatic Control, IEEE Transactions on 22 (5) (1977) 728-740.

[24] T. Yoshizawa, Stability theory by lyapunov's second method, The mathematical Society of Japan (9) (1966) 223.

[25] A. Isidori, Nonlinear control systems. 2 (1989), Vol. 2, Springer, 1989.

[26] L. Sidhom, M. Smaoui, D. Thomasset, X. Brun, E. Bideaux, et al., Adaptive higher order sliding modes for two-dimensional derivative estimation, in: Proceedings of the 18th IFAC World Congress, Vol. 18, 2011, pp. 3063-3071. 